BMJ

Open

Gastroenterology

\section{Menetrier's disease exacerbating ulcerative colitis and relieved by gastrectomy}

To cite: Kayes T, Bonnichsen M, Willmann L, et al. Menetrier's disease exacerbating ulcerative colitis and relieved by gastrectomy. BMJ Open Gastro 2021;8:e000806. doi:10.1136/ bmjgast-2021-000806

Received 14 0ctober 2021 Accepted 30 November 2021

Check for updates

(c) Author(s) (or their employer(s)) 2021. Re-use permitted under CC BY-NC. No commercial re-use. See rights and permissions. Published by BMJ.

${ }^{1}$ Department of

Gastroenterology and Hepatology, Liverpool Hospital, Liverpool, New South Wales, Australia

${ }^{2}$ Department of Upper Gl surgery, Liverpool Hospital, Liverpool, New South Wales, Australia

${ }^{3}$ Department of Medical Oncology, Liverpool Hospital, Liverpool, New South Wales, Australia

${ }^{4}$ Department of Rheumatology, Liverpool Hospital, Liverpool, New South Wales, Australia ${ }^{5}$ Department of Anatomical Pathology, Liverpool Hospital, Sydney, New South Wales, Australia

Correspondence to Dr Mark Bonnichsen; mbonnichsen@gmail.com

\section{ABSTRACT}

Ménétrier's disease (MD) is a rare gastropathy characterised by giant rugal folds which can present with nausea, vomiting, abdominal pain and protein losing gastropathy. We report a 21-year-old woman with comorbid MD and ulcerative colitis (UC). Management was complicated by limited treatment options for MD, significant symptom burden, worsening nutrition and difficulty determining which disease was the predominant cause of symptoms. Since age 18 the patient experienced recurrent UC flares characterised by diarrhoea, persistent vomiting and corticosteroid dependence. Endoscopic assessment demonstrated concurrent MD and active UC. 0ctreotide and cetuximab were trialled given persistent hypoalbuminaemia and suspicion for MD associated protein-losing gastropathy. UC management comprised dose-optimised infliximab and methotrexate. Repeat endoscopic assessment demonstrated improvement in UC without corresponding improvement in symptoms or hypoalbuminaemia. Nasojejunal feeding and parenteral nutrition failed to significantly improve nutritional status and accordingly the patient proceeded to radical total gastrectomy. Postoperatively, MD-associated symptoms and hypoalbuminemia resolved completely.

\section{CASE REPORT}

A 21-year-old woman presented for evaluation of ongoing diarrhoea and intermittent vomiting in the context of chronically active ulcerative pancolitis (UC). She had been diagnosed with $\mathrm{UC}$ at age 15 and initially managed with oral mesalazine granules and corticosteroids. From age 18, she had recurrent disease flares characterised by diarrhoea and vomiting, with associated corticosteroid dependence. After a trial of azathioprine was unsuccessful due to refractory vomiting, there was persistent hypoalbuminaemia with levels consistently $<28 \mathrm{~g} / \mathrm{L}$ which were attributed to her chronically active UC. Vedolizumab was commenced and subsequently dose escalated while corticosteroids were weaned. Concurrent endoscopic assessment for evaluation of vomiting demonstrated diffuse gastric nodularity and histology (figure 1) identified Menetrier's disease (MD) with characteristic foveolar hyperplasia and glandular atrophy. Gastric biopsies were negative for cytomegalovirus (CMV) and Helicobacter pylori infection. Despite 4-month treatment with monthly $20 \mathrm{mg}$ intramuscular injection of octreotide and a high protein diet, her symptoms persisted and serum albumin levels declined to $13 \mathrm{~g} / \mathrm{L}$.

At this point, while on fourweekly vedolizumab, her UC was reassessed with findings of a markedly elevated faecal calprotectin $(1300 \mathrm{ug} / \mathrm{g})$ and moderately severe pancolitis on colonoscopy (Ulcerative Colitis Endoscopic Index of Severity (UCEIS) score 4). Therapy was switched to combination infliximab and subcutaneous methotrexate $10 \mathrm{mg}$ weekly (subcutaneous route chosen to minimise nausea). Infliximab dosing was optimised using therapeutic drug monitoring (aiming for week 6 levels of $>15 \mathrm{mg} / \mathrm{L}$ and maintenance levels $\geq 10 \mathrm{mg} / \mathrm{L}$ ). Repeat endoscopic assessment at 6 weeks after commencement of infliximab showed endoscopic (UCEIS 3) and histological improvement in colitis. This corresponded with an improvement in lower gastrointestinal symptoms, however, nausea and vomiting persisted. Albumin levels remained low (15-20 g/L) and progress gastroscopy showed no macroscopic or microscopic improvement of MD. Throughout this period, infliximab levels were difficult to maintain at $10 \mathrm{mg} / \mathrm{L}$ despite aggressive infliximab dosing of up to $10 \mathrm{mg} /$ kg every 2 weeks.

Intravenous cetuximab, an epidermal growth factor receptor (EGFR) inhibitor, was trialled based on previous case reports of use in MD ( $750 \mathrm{mg}$ loading dose followed by $500 \mathrm{mg}$ weekly). ${ }^{1}$ After 2 weeks of treatment, there was no improvement in symptoms. She 


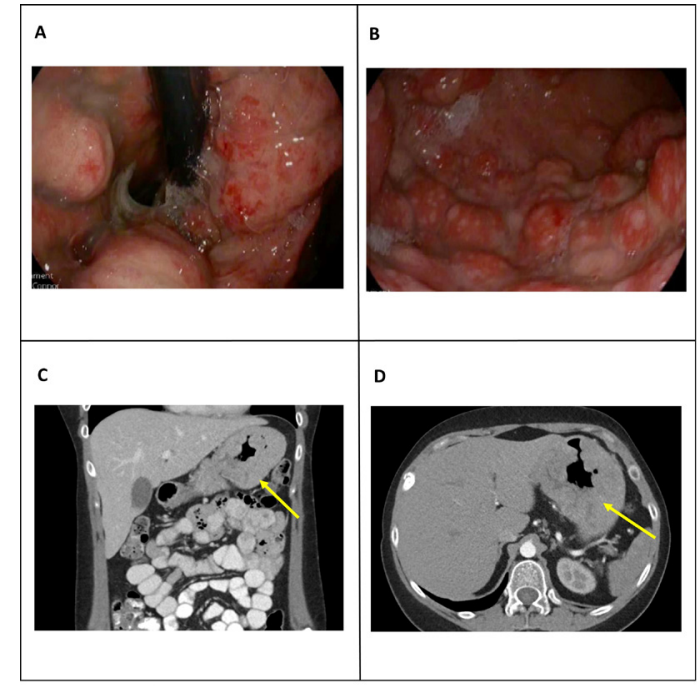

Figure 1 Endoscopic images of Menetrier's disease affecting the stomach (A-gastric fundus, B-gastric body) and abdominal CT (arterial phase) showing marked hypertrophy of the gastric rugae marked by arrow (coronal image-C, axial image-D).

also developed significant adverse reactions to cetuximab including myalgias, rigours and acneiform rash. Despite continuous nasojejunal feeding and total parenteral nutrition, nutritional status remained poor with severe refractory hypoalbuminaemia (maximum albumin reached was $16 \mathrm{~g} / \mathrm{L}$ ). Over this period, the patient developed significant weight $(13 \mathrm{~kg})$ secondary to fluid retention requiring commencement of diuretic therapy. The absence of response to medical therapy prompted the decision to consider gastrectomy.

The patient underwent a Radical Total Gastrectomy and Roux en-Y Hunt/Lawrence Pouch Reconstruction with lymph node dissection in case of any malignant changes. Histopathology demonstrated diffuse foveolar hyperplasia consistent with MD with no evidence of malignancy (figure 2). Postoperatively, all symptoms resolved completely. The patient also reported mood improvement postoperatively and she was able to return to tertiary studies and enjoy hobbies previously not possible due to persistent nausea and vomiting. Albumin levels quickly improved and remained persistently above $30 \mathrm{~g} / \mathrm{L}$ from day 14 post gastrectomy. Serum infliximab levels stabilised at $10 \mathrm{mg} / \mathrm{L}$ on fourweekly $(10 \mathrm{mg} / \mathrm{kg})$ infusions. Endoscopic assessment 8 weeks after gastrectomy showed a healthy esophagojejunal anastomosis and at 5 months of follow-up, her nausea remained absent. She experienced worsening diarrhoea and despite stable infliximab trough levels of $24-26 \mathrm{mg} / \mathrm{L}$, progress endoscopic assessment showed ongoing active colitis (UCEIS 3). Therapy was switched to Tofacitinib $10 \mathrm{mg}$ two times a day with good initial symptomatic response.

Postoperatively, the patient had the additional challenge of severe osteoporosis characterised by multiple atraumatic and painful vertebral fractures at the thoraco-lumbar spine. Bone mineral density testing via

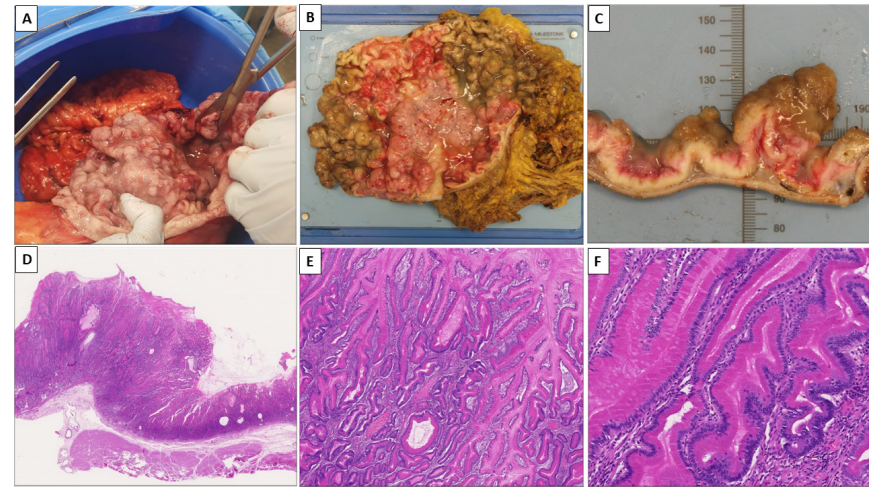

Figure 2 Macroscopic images of the gastrectomy specimen showing prominent gastric mucosal folds (A-intraoperative assessment of stomach mucosa, B-partially fixed opened stomach, $\mathrm{C}$-cross section across the nodular gastric wall). H\&E histopathological images showing features of MD disease $(D-$ whole slide image across all layers of the gastric wall showing marked mucosal thickening (10x), E-diffuse foveolar hyperplasia with elongated and occasionally dilated foveolar glands (50x), F-tortuous (corkscrew) foveolar glands with background of mild lamina propria chronic inflammation $(200 \times)$ ).

dual-energy X-ray absorptiometry showed $\mathrm{T}$ and $\mathrm{Z}$ scores of less than -4.0 SD at both lumbar spine and femoral neck. She was managed with vitamin D supplementation and teriparatide as initial anabolic therapy to reduce the risk of further fractures.

\section{DISCUSSION}

We present a rare case of MD with comorbid ulcerative pancolitis in a young woman requiring biologic therapy. The definitive diagnosis was delayed with endoscopic assessment up to twelve months prior to diagnosis noting prominent gastric folds, however, without confirmatory histology. Patient management was complicated due to limited evidence to guide management of MD, significant symptom burden, worsening hypoalbuminaemia and difficulty in ascertaining which disease, UC or MD, was predominantly responsible.

MD is a rare, acquired non-inflammatory condition characterised by aberrant EGFR signalling and transforming growth factor-a (TGF-a) overproduction in the stomach, leading to hyperplastic gastropathy and associated hyperproliferative protein losing gastropathy. ${ }^{2}$ Clinical manifestations include nausea, vomiting, abdominal pain with early satiety and resultant poor oral intake. ${ }^{12}$ Classic endoscopic features include antral sparing, giant oedematous rugae with histopathology revealing marked foveolar hyperplasia with tortuous glands. It is considered a premalignant disorder, associated with higher incidence of gastric cancer and consequent mortality. ${ }^{34}$ Literature describing MD is limited to case series and cohort studies and the aetiology remains unclear. It has been frequently associated with gastric CMV infection and occasionally postulated to relate to $H$. pylori infection. ${ }^{14}$ 
Comorbid Inflammatory bowel disease (IBD) with MD is very rare with only 14 patients previously reported in literature. ${ }^{2}$ In our case, MD significantly complicated the management of UC. We postulated that the protein-losing gastropathy caused by MD was the source of infliximab loss contributing to large fluctuations in drug levels and lack of clinical response with standard induction dosing. Based on this rationale, infliximab was aggressively dose-escalated with drug level monitoring and successful induction of clinical remission of UC. Supporting this, serum infliximab levels increased post gastrectomy, and were able to be maintained above therapeutic target with dose de-escalation from two to four weekly.

The severity of osteoporosis experienced by this patient is not common for young patients with IBD. The mechanism was thought to be multifactorial driven by chronic steroid use, vitamin D deficiency, systemic inflammatory illness and chronic illness related hypogonadism. Prior to gastrectomy the patient had persistent vitamin D deficiency (40-42 mmol/L) despite supplementation, which improved $(71 \mathrm{nmol} / \mathrm{L})$ postoperatively without further dose adjustment. Vitamin D deficiency from chronic malabsorption has been demonstrated in protein-losing enteropathies such as primary intestinal lymphangiec$\operatorname{tasia}^{5}$ and this may also contribute in MD.

As aforementioned, EGFR signalling has been implicated in the pathogenesis of MD. TGF- $\alpha$ increases gastric epithelial cell production and increased TGF- $\alpha$ immunoreactivity in abnormal regions of gastric mucosa has been demonstrated in patients with MD. Based on this, cetuximab has been proposed for management of MD and in a single-arm study, all seven patients reported symptom improvement within 1 week of starting cetuximab. ${ }^{1}$ Currently, there is no available guideline on the optimal duration of cetuximab therapy for remission of $\mathrm{MD}$, with some patients requiring treatment for up to 18 months and despite this still requiring gastrectomy. ${ }^{1}$ In our case, lack of response to cetuximab after a 2-week trial, emergence of new adverse effects, risk of gastric malignancy and patient preference were factors in the multidisciplinary consensus decision to proceed to gastrectomy.

\section{CONCLUSION}

We present a rare case of concomitant MD and UC in a young woman. Management was complicated due to difficulty in establishing which disease process was the primary cause for her symptoms. Overall, definitive treatment of her MD with total gastrectomy provided significant improvement in symptom burden and protein loss. Control of her UC, while currently improved, also remains a challenge.

Contributors TK, MB, LW: drafting of the article, critical revision of the article. AL, AD, CE-H, WengN, WatsonN, A-JW, DP, SJC: study concept and design, drafting of the article, critical revision of the article. ZH: histopathology slide preparation and manuscript remission. All authors have read and approved the final version. All authors have contributed to the paper and have not submitted the manuscript, in whole or in part, to other journals.

Funding The authors have not declared a specific grant for this research from any funding agency in the public, commercial or not-for-profit sectors.

Competing interests None declared.

Patient consent for publication Consent obtained directly from patient(s)

Ethics approval This study does not involve human participants.

Provenance and peer review Not commissioned; externally peer reviewed.

Data availability statement No data are available.

Open access This is an open access article distributed in accordance with the Creative Commons Attribution Non Commercial (CC BY-NC 4.0) license, which permits others to distribute, remix, adapt, build upon this work non-commercially, and license their derivative works on different terms, provided the original work is properly cited, appropriate credit is given, any changes made indicated, and the use is non-commercial. See: http://creativecommons.org/licenses/by-nc/4.0/.

\section{ORCID iDs}

Tahrima Kayes http://orcid.org/0000-0001-8927-3635

Mark Bonnichsen http://orcid.org/0000-0002-6697-3775

David Prince http://orcid.org/0000-0001-8087-0548

\section{REFERENCES}

1 Fiske WH, Tanksley J, Nam KT, et al. Efficacy of Cetuximab in the Treatment of Ménétrier's Disease. Sci Trans/ Med 2009;1:1-18.

2 Rao S, Viola A, Ksissa O, et al. Ménétrier's disease in a patient with refractory ulcerative colitis: a clinical challenge and review of the literature. BMJ Case Rep 2021:14:e246137.

3 Almazar AE, Penfield JD, Saito YA, et al. Survival times of patients with Menetrier's disease and risk of gastric cancer. Clin Gastroenterol Hepatol 2021;19:707-12.

4 Parianos C, Aggeli C, Sourla A, et al. Total gastrectomy for the treatment of Menetrier's disease persistent to medical therapy: a case report. Int J Surg Case Rep 2020;73:95-9.

5 Ozen A, Comrie WA, Ardy RC, et al. Cd55 deficiency, earlyonset protein-losing enteropathy, and thrombosis. N Engl J Med 2017;377:52-61. 